\title{
Analysis Tools to Uncover Variations in Picosecond Mode- Locked Pulses
}

\author{
Tushar Malica ${ }^{1}$, Jipeng Lin ${ }^{3}$, Thorsten Ackemann², Douglas J. Little ${ }^{1}$, Joshua P. Toomey ${ }^{1}$, Walter \\ Lubeigt $^{3}$, Nils Hempler ${ }^{3}$, and Deb M. Kane ${ }^{1 *}$ \\ ${ }^{1}$ Photonics Dynamical Systems Group, Department of Physics \& Astronomy, Macquarie University, Sydney 2109, Australia \\ ${ }^{2}$ Deaprtment of Physics, University of Strathclyde, John Anderson Building, Glasgow G4 ONG, Scotland, UK \\ ${ }^{3}$ M-Squared Ltd, 1 Kelvin Campus, West of Scotland Science Park, Glasgow G4 ONG, Scotland, UK \\ Author e-mail address: deb.kane@mq.edu.au
}

\begin{abstract}
A big data approach is demonstrated for quality assurance of mode-locked pulses longer than 20 ps. Real-time recordings of pulses sampled at $200 \mathrm{Gs} / \mathrm{s}$ are analysed to quantify pulse consistency within sequences of 50,000 pulses. (C) 2020 The Authors
\end{abstract}

Fundamental mode-locking in any laser system aims to produce a continuous sequence of pulses with (i) the shortest possible duration, (ii) an interpulse time interval that is exactly the roundtrip time of the laser cavity, and, (iii) for all the pulses to have the same pulse energy and peak amplitude. Quantifying the spread in these measures for pulses from a given mode-locked laser system requires a well-sampled measurement of the time series of a very large number of pulses. Such a measurement is possible using the current technology in fast real time digital sampling oscilloscopes, with the limitation that mode-locked pulses with a duration of 20 ps or more are required so they can be adequately sampled. The laser system, shown schematically in fig. 1, comprises an optically pumped vertical cavity surface emitting laser (VCSEL) operated in an extended cavity which also includes a semiconductor saturable absorber mirror (SESAM). Fundamental mode-locked operation has a pulse repetition frequency of $\sim 200 \mathrm{MHz}$. The wavelength is tunable, using a birefringent filter, through about $10 \mathrm{~nm}$ about a centre wavelength of $\sim 989 \mathrm{~nm}$. Mode-locked pulses with a minimum duration of about $20 \mathrm{ps}$ are obtained [1, 2]. This system was selected as being a suitable laser system to collect the long time series output power data required to investigate the statistics of the variation in the pulses. This system has been shown to generate a range of different dynamical outputs which have been mapped and reported in [3]. Here-in we interrogate the consistency of the pulses in the fundamental mode-locking regime of operation using several measurements. These include conventional statistical measures of average values and their associated standard deviations for the pulse duration, the interpulse interval, and the pulse amplitude. Additionally, these quantities are contrasted with permutation entropy (PE) calculations as a way of testing whether PE can be a useful measure of pulse quality for a given mode-locked laser. PE combines the concept of symbolic dynamics with that of entropy to directly account for temporal information in a time series [4]. It is a measure of time series complexity based on the entropy of ordinal permutations or patterns. Its use to interrogate amplitude jitter and timing jitter in pulsed output from two different laser systems was demonstrated in [5]. Neither of the laser systems studied in [5] was regarded as a source of high-quality laser pulses.

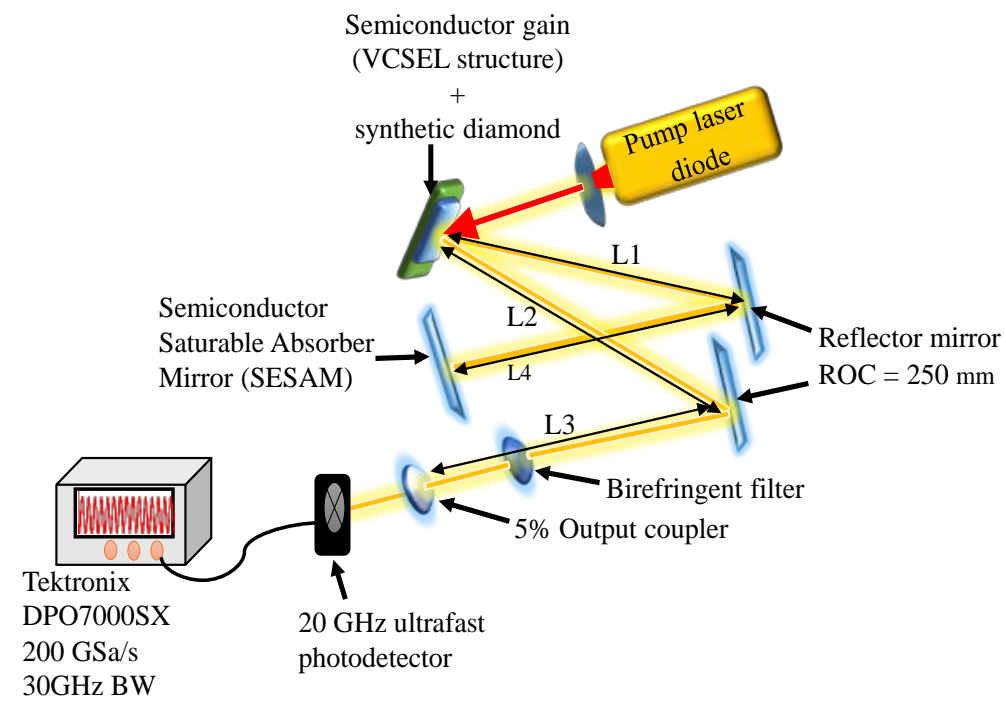

Fig. 1. Experimental laser system for passive mode-locking using an optically pumped VCSEL semiconductor gain device and a SESAM in an extended cavity - a VECSEL system. ROC- radius of curvature. BW - bandwidth. Reproduced with permission from [3]. 
The fundamental mode-locked pulses from the VECSEL system were sampled with a $20 \mathrm{GHz}$ ultrafast-20MM photodetector (Advanced Laser Diode Systems) connected to a Tektronix DPO7000SX oscilloscope using 200 $\mathrm{GSa} / \mathrm{s}$ sampling rate. A $250 \mu$ s output time series containing $\sim 50 \times 10^{6}$ data points collected at 5 ps intervals was collected at each operating point - wavelength steps of $1 \mathrm{~nm}$ (with one of $0.5 \mathrm{~nm}$ at $994.5 \mathrm{~nm}$ ), and pump laser diode currents ranging from $24 \mathrm{~A}$ to $18 \mathrm{~A}$ at intervals of $1 \mathrm{~A}$. The subset of operating points for which fundamental mode-locked output was obtained are presented in [3]. The time series for all these conditions were further analyzed to test the pulse quality using the statistical measures and permutation entropy. Fig. 2 shows the strong correlation between three of the measurands investigated - the residual permutation entropy ( 1 minus the permutation entropy value), analysed with a delay time of the roundtrip time; the standard deviation in the interpulse period and the variation in the amplitude of the pulses expressed as a percentage of the average amplitude. The operating condition for fig 2 (current to the $808 \mathrm{~nm}$ pump laser of $23 \mathrm{~A}$ ) is one where the pulses have lower overall quality compared to conditions which give the shortest duration, most consistent pulses. It is the current at which the highest average power is achieved for fundamental mode-locked output. Results from the full set of operating conditions will be presented. Overall the approach is shown to be sound. The operating points that give the most consistent pulses are those with the shortest duration. This reinforces the usual experimental approach to optimizing a mode-locked laser system. However, it is also useful to know qualitative and quantitative trends in how the pulse quality changes around the optimum operating conditions, especially when the system may be intended to be wavelength tunable.

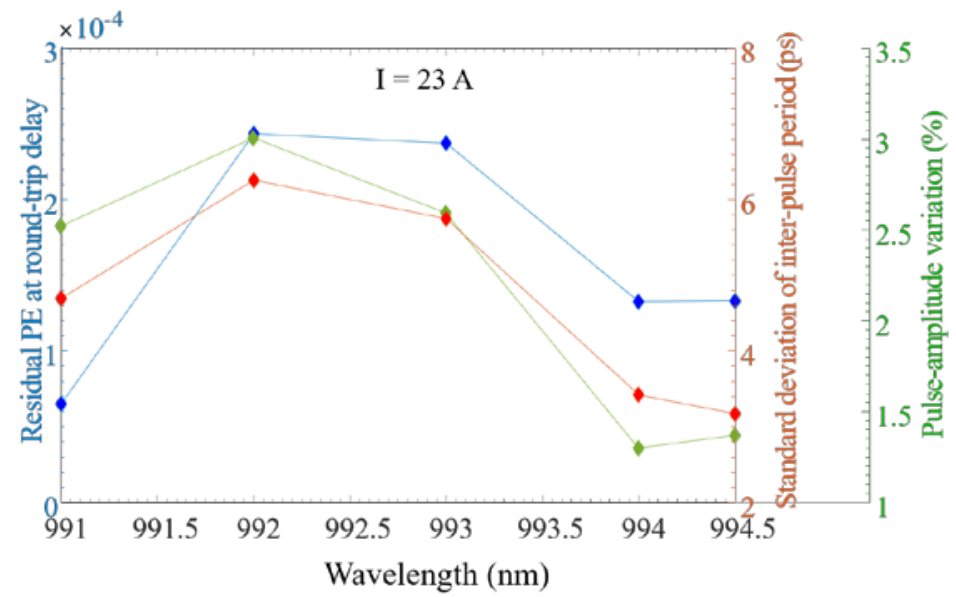

Fig. 2. Residual permutation entropy (PE) calculated using a time step of the roundtrip time for the laser system ( $\sim 5.1 \mathrm{~ns}$ ), standard deviation of the inter-pulse period of $\sim 5.1 \mathrm{~ns}$, and, pulse amplitude variation expressed as a percentage relative to the average pulse amplitude. Note the average power is $0.96 \mathrm{~W}$ at $991 \mathrm{~nm}, 0.86 \mathrm{~W}$ at $993 \mathrm{~nm}$, and 0.6 W at $994.5 \mathrm{~nm}$ [3].

\section{References}

[1] N. Hempler, B. Bialkowski, C. J. Hamilton, G. T. Maker, and G. P. A. Malcolm, "Development and commercialization of mode-locked VECSELs,” Proc. SPIE 9349, 93490K (2015).

[2] N. Hempler, W. Lubeigt, B. Bialkowski, C. J. Hamilton, G.T. Maker, and G. P. A. Malcolm, "Advances in commercial, mode-locked vertical external cavity surface emitting lasers", Proc. SPIE 9734, 97340X (2016).

[3] T. Malica, J. Lin, T. Ackemann, D. J. Little, J. P. Toomey, D. Paboeuf, W. Lubeigt, N. Hempler, G. Malcolm, G. T. Maker, and D. M. Kane, 'Mapping the dynamical regimes of a SESAM mode-locked VECSEL with a long cavity using time series analysis”, Optics Express, 26(13), 16624-16638 (2018).

[4] C. Bandt and B. Pompe, “Permutation entropy: A natural complexity measure for time series”, Phys. Rev. Lett. 88, 174102. (2002).

[5] J P Toomey, D M Kane and T Ackemann, “Complexity in pulsed nonlinear laser systems interrogated by permutation entropy”, Optics Express 22(15), 17840-17853 (2014). 